\title{
Smooth Periodic Solutions with Equal Period for KP-MEW $(2,2)$ Equation
}

\author{
Minzhi Wei, Liping He* \\ School of Information and Statistics, Guangxi University of Finance and Economics, Nanning, China \\ Email: *help0613@126.com
}

How to cite this paper: Wei, M.Z. and He, L.P. (2021) Smooth Periodic Solutions with Equal Period for KP-MEW $(2,2)$ Equation. Journal of Applied Mathematics and Physics, 9, 1515-1521.

https://doi.org/10.4236/jamp.2021.97103

Received: June 24, 2021

Accepted: July 12, 2021

Published: July 15, 2021

Copyright (อ 2021 by author(s) and Scientific Research Publishing Inc. This work is licensed under the Creative Commons Attribution International License (CC BY 4.0).

http://creativecommons.org/licenses/by/4.0/

\begin{abstract}
In this paper, the KP-MEW $(2,2)$ equation is considered under a certain parametric condition. We prove that the equation has two isochronous centers under certain parametric conditions, and there exist two families of periodic solutions with equal period.
\end{abstract}

\section{Keywords}

KP-MEW(2,2) Equation, Abelian Integral, Picard-Fuchs Equation, Equal Period

\section{Introduction}

The KdV equation [1]

$$
q_{t}+a q q_{x}+q_{x x x}=0
$$

is a model that governs the one-dimensional propagation of small-amplitude, weakly dispersive waves. The nonlinear term $q q_{x}$ and the linear dispersion term $q_{x x x}$ in Equation (1.1) cause the steepening of wave form and the spread of the wave, respectively. After that, one of the well known 2-dimensional generalizations of the KdV equation is KP equation [2] which was derived:

$$
q_{t}+a q q_{x}+q_{x x x}+q_{y y}=0,
$$

More recently, MEW equation and KP-MEW equation were given and investigated on some methods [3] [4] [5]. Particularly, Asit Saha [6] considered the generalized KP-MEW equation

$$
\left(q_{t} \pm\left(q^{m}\right)_{x} \pm\left(q^{n}\right)_{x x t}\right)_{x} \pm q_{y y}=0
$$

by using the theory of bifurcations of planar dynamical systems [7]. More precisely, for $m=n=2$, what called KP-MEW $(2,2)$ equation in the form

$$
\left(q_{t}+\left(q^{2}\right)_{x}+\left(q^{2}\right)_{x x t}\right)_{x}+q_{y y}=0
$$


is investigated by Li and Song [8] by bifurcations method to find compacton-like wave and a kink-like wave for (1.4) when integral constant $g$ was not neglected. After that, (1.4) was investigated to find the peakon soliton, cuspon soliton and smooth soliton solutions on the boundary condition by using the phase portrait analytical technique [9].

In presented paper, we consider the $\operatorname{KP}-\operatorname{MEW}(2,2)$ equation in the form

$$
\left(q_{t}-\left(q^{2}\right)_{x}-\left(q^{2}\right)_{x x t}\right)_{x}-q_{y y}=0,
$$

where integral constant $g<0, c=-1$. As the relationship between wave speed and period is significant [10] [11], we prove that the $\operatorname{KP}-\operatorname{MEW}(2,2)$ equation has two isochronous centers under certain parameter conditions. Consequently, we find that there exist two families of periodic solutions with equal period.

This paper is organized as follows. In Sec. 2, for parameters $c=-1$ and $g<0$, the phase portraits of systems (2.2) are shown. In Sec. 3, we prove that the KP-MEW $(2,2)$ equation has two isochronous centers under certain parameter conditions and there exist two families of periodic solutions with equal period.

\section{Phase Portrait}

Making the transformations $q(x, t)=q(x-c t)=u(\xi)$ to (1.5), integrating it twice, it arrives to

$$
-(1+c) u-u^{2}+c\left(u^{2}\right)^{\prime \prime}=g,
$$

where $c$ is the wave speed, $g$ is the integral constant, 'is the derivative with respect to $\xi$.

Equation (2.1) is equivalent to the planar dynamical system

$$
\left\{\begin{array}{l}
\frac{\mathrm{d} u}{\mathrm{~d} \xi}=y, \\
\frac{\mathrm{d} y}{\mathrm{~d} \xi}=\frac{g+(1+c) u+u^{2}-2 c y^{2}}{2 c u} .
\end{array}\right.
$$

Using the "timescale" transformation $\mathrm{d} \xi=2 c u \mathrm{~d} \tau,(2.2)$ reduces to the regular system

$$
\left\{\begin{array}{l}
\frac{\mathrm{d} u}{\mathrm{~d} \tau}=2 c u y, \\
\frac{\mathrm{d} y}{\mathrm{~d} \tau}=g+(1+c) u+u^{2}-2 c y^{2},
\end{array}\right.
$$

with the first integral

$$
H(u, y)=c u^{2} y^{2}-\left(\frac{g}{2} u^{2}+\frac{1+c}{3} u^{3}+\frac{1}{4} u^{4}\right)=h,
$$

where $h$ is an integral constant. Thus, systems (2.2) and (2.3) have the same topological phase portraits except for the straight line $u=0$. Under some parametric conditions, the variable $\tau$ is a fast variable while the variable $\xi$ is a slow variable in the sense of the geometric singular perturbation theory [12]. 
For the given constants $c=-1$ and $g<0$, the phase portrait is shown in Figure 1.

From Figure 1, we know that on the condition $c=-1, g<0$, there are two family of periodic orbits for system (1.5), linking with the theory of dynamic systems, the periodic solutions correspond to the periodic orbits. Each periodic orbit $\Gamma_{h}$ is contained in a unique level set $(u, y): H(u, y)=h$ and its period equals to

$$
T(h)=\oint_{\Gamma_{h}} \mathrm{~d} \xi=\oint_{\Gamma_{h}} \frac{\mathrm{d} \phi}{y} .
$$

The function $T(h)$ is called periodic function. If all orbits around the center have the same period, then the center is isochronous.

\section{Periodic Solutions with Equal Period}

In order to express $T(h)$ as a linear combination of Abelian integrals, we define a series of functions

$$
J_{i}(h)=\oint_{\Gamma_{h}}(-2) u^{i+1} y \mathrm{~d} u,
$$

then

$$
\begin{aligned}
J_{i}^{\prime}(h) & =\oint_{\Gamma_{h}}(-2) u^{i+1} \frac{\partial y}{\partial h} \mathrm{~d} u \\
& =\oint_{\Gamma_{h}}(-2) u^{i+1} \frac{1}{-2 u^{2} y} \mathrm{~d} u \\
& =\oint_{\Gamma_{h}} \frac{u^{i-1}}{y} \mathrm{~d} u
\end{aligned}
$$

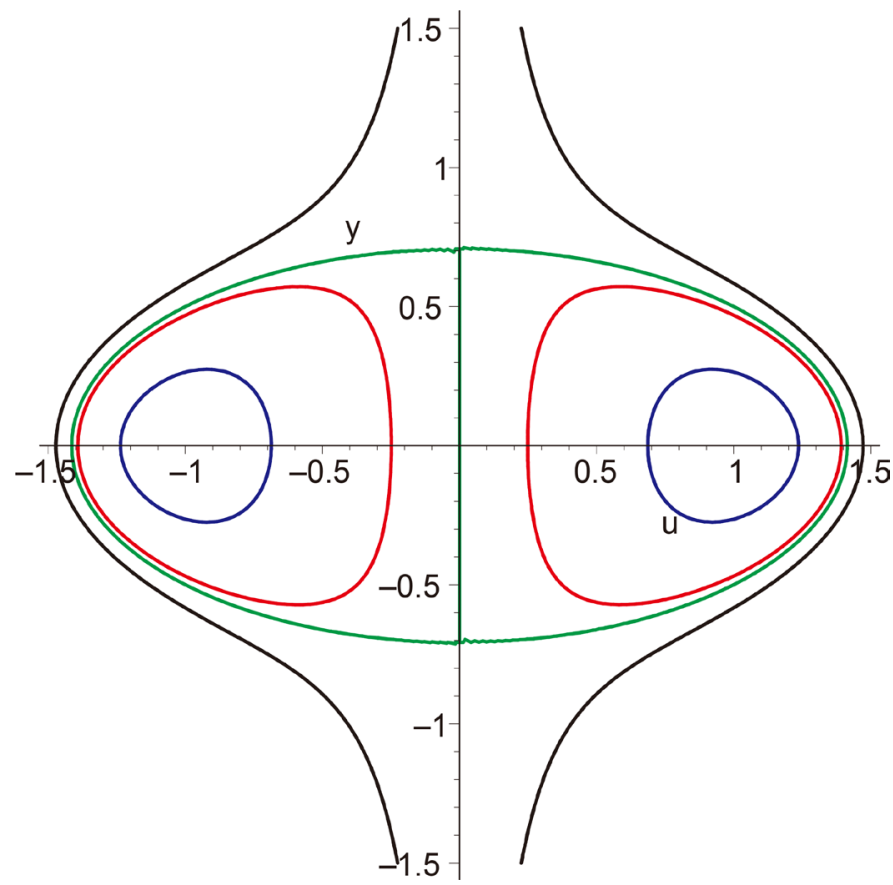

Figure 1. Phase portrait on the condition $c=-1, g<0$. 
where 'is the derivative with respect to $h$. Therefore, it has $J_{1}^{\prime}(h)=T(h)$.

Combining Equatioins (3.1) with (2.4) and quoting to [13], it has

$$
\begin{aligned}
J_{i}(h) & =\oint_{\Gamma_{h}}(-2) u^{i+1} y \mathrm{~d} u=\oint_{\Gamma_{h}} \frac{-2 u^{i+1} y^{2}}{y} \mathrm{~d} u \\
& =\oint_{\Gamma_{h}} \frac{-2 u^{i+1}}{y}\left[\frac{g}{2}-\frac{1}{4} u^{2}+h u^{-2}\right] \mathrm{d} u \\
& =g J_{i+2}^{\prime}(h)+\frac{1}{2} J_{i+4}^{\prime}(h)+2 h J_{i}^{\prime}(h),
\end{aligned}
$$

that is

$$
J_{i}(h)=g J_{i+2}^{\prime}(h)+\frac{1}{2} J_{i+4}^{\prime}(h)+2 h J_{i}^{\prime}(h) .
$$

And integration parts, $J_{i}(h)$ has another expression:

$$
J_{i}(h)=\oint_{\Gamma_{h}}(-2) u^{i+1} y \mathrm{~d} u=\oint_{\Gamma_{h}} 2 \frac{u^{i+2}}{i+2} \mathrm{~d} y,
$$

linking with (3.2) and (2.4), it becomes

$$
\begin{aligned}
J_{i}(h) & =\oint_{\Gamma_{h}} 2 \frac{u^{i+2}}{(i+2) y}\left(-\frac{1}{4} u+h u^{-3}\right) \mathrm{d} u \\
& =-\frac{1}{i+2}\left[\frac{1}{2} J_{i+4}^{\prime}(h)-2 h J_{i}^{\prime}(h)\right]
\end{aligned}
$$

then we obtain

$$
(i+2) J_{i}(h)=-\frac{1}{2} J_{i+4}^{\prime}(h)+2 h J_{i}^{\prime}(h) .
$$

Setting $i=-1,0,1,2$, we obtain the vector $J(h)=\operatorname{col}\left(J_{1}(h), J_{0}(h), J_{1}(h), J_{2}(h)\right)$ satisfies the following Picard-Fuchs equation:

$$
\left\{\begin{array}{l}
J_{-1}(h)=\frac{1}{2} J_{3}^{\prime}(h)+g J_{1}^{\prime}(h)+2 h J_{-1}^{\prime}(h), \\
J_{0}(h)=\frac{1}{2} J_{4}^{\prime}(h)+g J_{2}^{\prime}(h)+2 h J_{0}^{\prime}(h), \\
J_{1}(h)=\frac{1}{2} J_{5}^{\prime}(h)+g J_{3}^{\prime}(h)+2 h J_{1}^{\prime}(h), \\
J_{2}(h)=\frac{1}{2} J_{6}^{\prime}(h)+g J_{4}^{\prime}(h)+2 h J_{2}^{\prime}(h), \\
J_{-1}(h)=-\frac{1}{2} J_{3}^{\prime}(h)+2 h J_{-1}^{\prime}(h), \\
2 J_{0}(h)=-\frac{1}{2} J_{4}^{\prime}(h)+2 h J_{0}^{\prime}(h), \\
3 J_{1}(h)=-\frac{1}{2} J_{5}^{\prime}(h)+2 h J_{1}^{\prime}(h), \\
4 J_{2}(h)=-\frac{1}{2} J_{6}^{\prime}(h)+2 h J_{2}^{\prime}(h) .
\end{array}\right.
$$

It is a system of linear equations with respect to $J_{1}(h), J_{0}(h), J_{1}(h), J_{2}(h)$ and $J_{i}^{\prime}(h), i=-1, \cdots, 6$. After doing a series of complicated calculations, we find 
they hold the relationships:

$$
\begin{gathered}
J_{-1}(h)=2 h J_{-1}^{\prime}(h)+\frac{g}{2} J_{1}^{\prime}(h), \\
J_{0}(h)=\frac{4 h}{3} J_{0}^{\prime}(h)+\frac{g}{3} J_{2}^{\prime}(h), \\
J_{1}(h)=\left(h-\frac{g^{2}}{4}\right) J_{1}^{\prime}(h), \\
J_{2}(h)=-\frac{4 h}{3} J_{0}^{\prime}(h)+\left(\frac{4 h}{5}-\frac{4 g^{2}}{15}\right) J_{2}^{\prime}(h) .
\end{gathered}
$$

Combining (3.5)-(3.8), we can obtain Lemma 3.1 as follows:

Lemma 3.1. The vector $J(h)=\operatorname{col}\left(J_{1}(h), J_{0}(h), J_{1}(h), J_{2}(h)\right)$ satisfies the following Picard-Fuchs equation:

$$
\left(\begin{array}{c}
J_{-1}(h) \\
J_{0}(h) \\
J_{1}(h) \\
J_{2}(h)
\end{array}\right)=\left(\begin{array}{cccc}
2 h & 0 & \frac{g}{2} & \frac{g}{3} \\
0 & \frac{4 h}{3} & 0 & 0 \\
0 & 0 & h-\frac{g^{2}}{4} & 0 \\
0 & -\frac{4 h}{3} & 0 & \frac{4 h}{5}-\frac{4 g^{2}}{15}
\end{array}\right)\left(\begin{array}{c}
J_{-1}^{\prime}(h) \\
J_{0}^{\prime}(h) \\
J_{1}^{\prime}(h) \\
J_{2}^{\prime}(h)
\end{array}\right) .
$$

As the result shown above, $J_{1}(h)=\left(h-\frac{g^{2}}{4}\right) J_{1}^{\prime}(h)$, differentiating both sides of the equation, we have

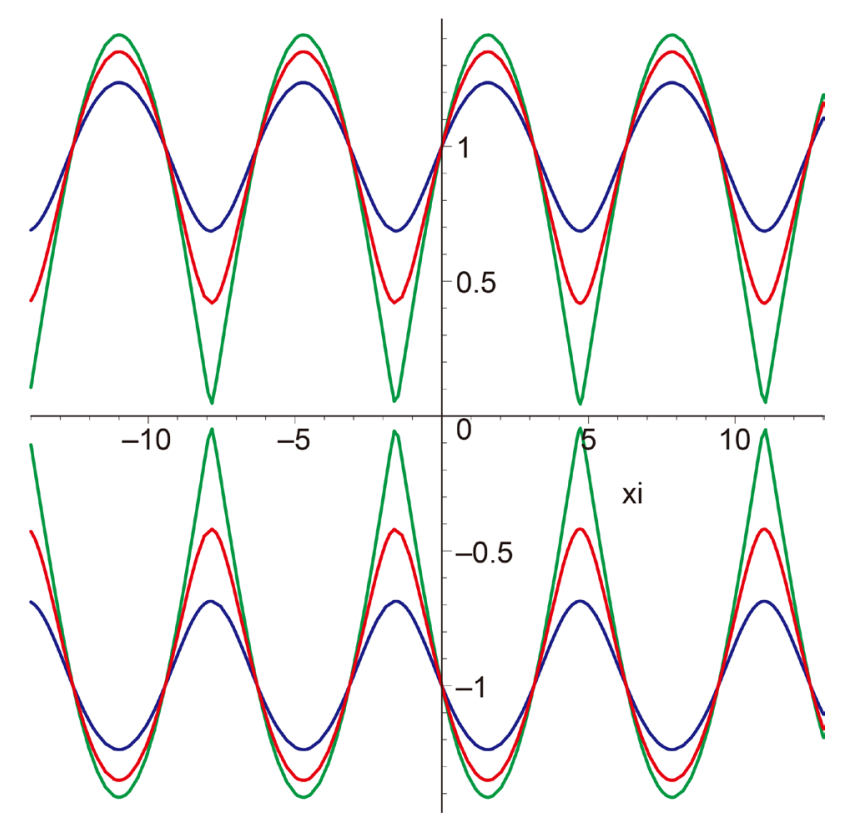

Figure 2. Simulations of two families of periodic solutions with the equal period. Setting $h=0.001,0.08,0.18$, they give rise to the green wave, red wave and the blue wave, respectively. 


$$
J_{1}^{\prime}(h)=J_{1}^{\prime}(h)+\left(h-\frac{g^{2}}{4}\right) J_{1}^{\prime \prime}(h)
$$

hence, $\left(h-\frac{g^{2}}{4}\right) J_{1}^{\prime \prime}(h)=0$, it is said that $J_{1}^{\prime \prime}(h)=0$. It can be concluded that $J_{1}^{\prime}(h)=C$, with $C$ is a constant, it implies $T(h)=C$. Therefore, it is obtained Theorem 3.1 .

Theorem 3.1. If $c=-1, g<0$, the system (1.5) has two isochronous centers at $E_{1}\left(\frac{-(1+c)+\sqrt{\Delta}}{2}, 0\right)$ and $E_{2}\left(\frac{-(1+c)-\sqrt{\Delta}}{2}, 0\right)$. Consequently, Equation (1.5) has two families of periodic solutions with the equal period, see Figure 2.

\section{Conclusion}

In the present paper, we prove that the planar dynamical system has two isochronous centers under certain parameter conditions by using Picard-Fuchs equation, it is said that there exist two families of periodic solutions with equal period.

\section{Acknowledgements}

This work is supported by National Natural Science Foundation of Guangxi Province (2020JJB110007), Guangxi College Enhancing Youths Capacity Project (2020KY16019, 2020KY16020).

\section{Conflicts of Interest}

The authors declare no conflicts of interest regarding the publication of this paper.

\section{References}

[1] Korteweg, D.J. and Vries, G.D. (1895) On the Change of Form of Long Waves Advancing in a Rectangular Channel, and a New Type of Long Stationary Waves. Philosophical Magazine, 39, 422-443. https://doi.org/10.1080/14786449508620739

[2] Kadomtsev, B.B. and Petviashvili, V.I. (1974) On the Stability of Solitary Waves in Weakly Dispersive Media. Soviet Physics-JETP, 39, 285-295.

[3] Esen, A. (2006) A Lumped Galerkin Method for the Numerical Solution of the Modified Equal-Width Equation Using Quadratic B-Splines. International Journal of Computer Mathematics, 83, 449-459. https://doi.org/10.1080/00207160600909918

[4] Esen, A. and Kutluay, S. (2008) Solitary Wave Solutions of the Modified Equal Width Wave Equation. Communications in Nonlinear Science and Numerical Simulation, 13, 1538-1546. https://doi.org/10.1016/j.cnsns.2006.09.018

[5] Wazwaz, A.M. (2005) The Tanh Method and the Sine-Cosine Method for Solving the KP-MEW Equation. International Journal of Computer Mathematics, 82, 235-246. https://doi.org/10.1080/00207160412331296706

[6] Saha, A. (2012) Bifurcation of Travelling Wave Solutions for the Generalized KP-MEW Equations. Communications in Nonlinear Science and Numerical Simu- 
lation, 17, 3539-3551. https://doi.org/10.1016/j.cnsns.2012.01.005

[7] Li, J.B. (2013) Singular Nonlinear Traveling Wave Equations: Bifurcation and Exact Solutions. Science, Beijing.

[8] Li, S.Y. and Song, M. (2014) Compacton-Like Wave and Kink-Like Wave Solutions of the Generalized KP-MEW $(2,2)$ Equation. Physica Scripta, 89, Article ID: 035202. https://doi.org/10.1088/0031-8949/89/03/035202

[9] Wei, M.Z., Tang, S.Q., Fu, H.L. and Chen, G.X. (2013) Single Peak Solitary Wave Solutions for the Generalized KP-MEW $(2,2)$ Equation under Boundary Condition. Applied Mathematics and Computation, 219, 8979-8990. https://doi.org/10.1016/j.amc.2013.03.007

[10] Chen, A.Y., Tian, C.X. and Huang, W.T. (2018) Periodic Solutions with Equal Period for the Friedmann-Robertson-Walker Model. Applied Mathematics Letters, 77, 101-107. https://doi.org/10.1016/j.aml.2017.10.007

[11] Chen, A.Y., He, X.K. and Tian, C.X. (2019) Isochronous Cosmological Solutions of the Friedmann Robertson Walker Model. Modern Physics Letters A, 11, Article ID: 1950062. https://doi.org/10.1142/S0217732319500627

[12] Yan, W.F., Liu, Z.R. and Liang, Y. (2014) Existence of Solitary Waves and Periodic Waves to a Perturbed Generalized KdV Equation. Mathematical Modelling and Analysis, 4, 537-555. https://doi.org/10.3846/13926292.2014.960016

[13] Zhao, Y.L. (2005) On the Monotonicity of the Period Function of a Quadratic System. Discrete and Continuous Dynamical Systems Journal, 13, 795-810. https://doi.org/10.3934/dcds.2005.13.795 